Discussion Paper No. 657

\title{
CONSISTENCY AND UNANIMITY \\ IN THE HOUSE ALLOCATION PROBLEMS I: COLLECTIVE INITIAL ENDOWMENTS
}

\author{
Koji Takamiya
}

March 2006

The Institute of Social and Economic Research Osaka University

6-1 Mihogaoka, Ibaraki, Osaka 567-0047, Japan 


\title{
Consistency and unanimity in the house allocation problems I: Collective initial endowments*
}

\author{
Koji TAKAMIYA ${ }^{\dagger}$ \\ Institute of Social and Economic Research, \\ Osaka University
}

Preliminary version, March 162006

\begin{abstract}
This paper studies allocation correspondences in the house allocation problems with collective initial endowments. We examine the implications of two axioms, namely "consistency" and "unanimity." Consistency requires the allocation correspondence be invariant under reductions of population. Unanimity requires the allocation correspondence respect unanimity, that is, it assigns to every agent the object that ranks best for him whenever possible. We prove that if an allocation correspondence satisfies these two axioms, then it is a subcorrespondence of the Pareto correspondence. Further, we give a characterization of the Pareto correspondence using a version of "converse consistency."
\end{abstract}

JEL Classification - C78, D70.

Keywords - house allocation problem, axiomatic characterization, consistency, unanimity, Pareto efficiency.

\footnotetext{
${ }^{*}$ All errors are my own responsibility.

${ }^{\dagger}$ Institute of Social and Economic Research, Osaka University. 6-1 Mihogaoka Ibaraki Osaka 567-0047 JAPAN. tel: +81-6-6879-8556. E-mail: takamiya@iser.osaka-u.ac.jp.
} 


\section{Introduction}

\subsection{Background}

This paper studies allocation correspondences for house allocation problems. In particular, we study the case of collective initial endowments, i.e. no objects are initially owned by agents. In a house allocation problem, there is a set of finitely many agents, and a set of indivisible objects. The objects are initially owned collectively by the set of agents, and to be allocated to the agents so that each agent receives exactly one object. Each agent has a preference over the set of objects. This kind of problems arise in various real life situations in which monetary compensation is not feasible. Since the problem has been first considered by Shapley and Scarf (1974), numerous papers have studied this theme. In particular, a growing number of papers have appeared in recent years.

The purpose of this study is to examine properties of allocation correspondences that satisfy two axioms, namely "consistency" and "unanimity."

"Consistency" is a unifying property that is used many axiomatic studies of game-theoretic models of resource allocation. It is worth noting that many allocation rules in various models are characterized using consistency. (For survey, Thomson 1996, 1998.) Consistency basically requires the allocation rule be "invariant" to reduction of population: To be illustrative, consider a group of agents facing an allocation problem and some "allocation method" with which we want to solve this problem. Let us apply this allocation method to the problem, and obtain an allocation $x$. Then suppose that some of the agents have left the scene, and the remaining agents faces the "same" problem again, which is called the "reduced" problem. Now the allocation method being "consistent" means that applying the allocation method to the "reduced" problem again yields the allocation $x$ restricted to the remaining group. In defining consistency for each specific environment, it is essential how to define "reduced" problems. In the case of the present environment, there is the natural definition due to Ergin (2000), which we will use in this paper, too.

"Unanimity" is simple and uncontroversial principle in collective decision making in general. The axiom requires the rule adopt those choices (whenever available) which is unanimously approved by the agents. This seems a minimum requirement in terms of collective welfare. In the present context, unanimity is implied by Pareto efficiency .

Consistent allocation correspondences (and single-valued rules, too) for this environment has first considered by Ergin (2000), and subsequently studied by Ehlers and Klaus (2005) and others. In the previous studies, Pareto efficiency is a prevalent axiom. To our knowledge, however, no authors have ever explicitly considered unanimity or similar axioms in this context.

\section{$0.2 \quad$ Results}

As the main theorem, we prove that if an allocation correspondence is consistent and unanimous, then the correspondence is a subcorrespondence of the Pareto correspondence. Since the Pareto correspondence itself is consistent and unani- 
mous, the Pareto correspondence is the unique maximal correspondence that satisfies these two axioms. In the previous studies of consistency in this environment, Pareto efficiency constantly has been considered as an axiom. That is, they require allocation correspondences to be Pareto efficient, and examine its consequences together with other axioms. In contrast, the present study obtains Pareto efficiency as a result, rather than requiring it. By this result, all the foregoing characterization using consistency and Pareto efficiency simultaneously (such as Ergin (2000), and Ehlers and Klaus (2005)) can be strengthen by replacing Pareto efficiency with unanimity.

Further, we provide an axiomatic characterization of the Pareto correspondence using a version of "converse consistency" (which is different from the version in the previous studies of this model) together with the two axioms. It is worth noting that although the Pareto correspondence has been treated as one of important concepts, there has not been axiomatic support to the correspondence itself.

The present study exclusively deals with the case of collective initial endowments. In the companion piece (Takamiya, 2006), however, we are dealing with the case of private initial endowments. There, we introduce an extended definition of house allocation problems with private endowments that allows a natural operation of population reduction. And we show that consistency and unanimity, the counterpart of the axioms as considered in the present work, characterizes the core correspondence. We suppose that it is of some interest to compare the results in the cases of collective and individual initial endowments.

This paper is organized as follows: Section 1 provides preliminaries. Section 2 states the results. And Section 3 gives the proofs of the theorems.

\section{Preliminaries}

Let $\mathcal{N}$ be the set of potential agents. And let $\mathcal{H}$ be the set of objects. A house allocation problem (simply, problem, henceforth) is a list $\mathcal{E}=(N, H, R)$. Here, $N \subset \mathcal{N}, N \neq \emptyset$, and $H \subset \mathcal{H}, H \neq \emptyset$ such that $\# N=\# H$. And $R:=\left(R^{i}\right)_{i \in N}$ is a preference profile. Assume each $R^{i}$ is a linear ordering ("a ranking with no ties") over $H$. That is, $R^{i}$ is a complete, transitive and anti-symmetric binary relation over $H$. As usual, $P^{i}$ ( $I^{i}$, resp.) denotes the asymmetric (symmetric, resp.) part of $R^{i}$. For $H^{\prime} \subset H, \max R^{i}\left(H^{\prime}\right)$ denote the element $x \in H^{\prime}$ such that for any $y \in H^{\prime}$, $x R^{i} y$.

Let a problem $\mathcal{E}=(N, H, R)$ be given. An allocation $x$ is a bijection from $N$ onto $H . x(i)$ denotes the object allocated to $i$. Let $A(\mathcal{E})$ denote the set of allocations in problem $\mathcal{E}$. Let $x$ and $y$ be allocations. And let $R$ be a preference profile. $x$ Pareto dominates $y$ under $R$ if for each $i \in N, x(i) R^{i} y(i)$, and there exists some $j \in N$ such that $x(j) P^{j} y(j) . \quad x$ is Pareto efficient under $R$ if no allocation Pareto dominates $x$ under $R$.

An allocation correspondence (or correspondence, henceforth) is a multivalued function which associates each problem with a set of allocations in that problem. Let $\varphi$ denote a correspondence. The Pareto correspondence is the correspondence that specifies the set of Pareto efficient allocations for each problem. 
Let us denote the Pareto correspondence by $\mathcal{P}$.

Let $\mathcal{U}^{0}$ denote the correspondence such that for any $\mathcal{E}=(N, H, R)$,

$$
\mathcal{U}^{0}(\mathcal{E}):=\left\{x \mid \forall i \in N, x(i)=\max R^{i}(H)\right\} .
$$

$\varphi$ is unanimous if for every problem $\mathcal{E}=(N, H, R), \varphi(\mathcal{E})=\mathcal{U}^{0}(\mathcal{E})$ if $\mathcal{U}^{0}(\mathcal{E})$ is nonempty.

Let a problem $\mathcal{E}=(N, H, R)$ be given. Let $N^{\prime} \subset N\left(N^{\prime} \neq \emptyset\right)$ and $x \in A(\mathcal{E})$. A reduced problem of $\mathcal{E}$ relative to $N^{\prime}$ and $x$ is a problem $r_{\left(N^{\prime}, x\right)}(\mathcal{E})=\left(N^{\prime}, H^{\prime}, R^{\prime}\right)$. Here $H^{\prime}=x\left(N^{\prime}\right)$ and $R^{\prime}=\left(R^{\prime i}\right)_{i \in N^{\prime}}$, where $R^{\prime i}=\left.R^{i}\right|_{H^{\prime}}$ for each $i \in N^{\prime} . \varphi$ is consistent if for every problem $\mathcal{E}=(N, H, R)$, and every $N^{\prime} \subset N\left(N^{\prime} \neq \emptyset\right)$ and $x \in A(\mathcal{E})$, it is satisfied that $x \in \varphi(\mathcal{E})$ implies $\left.x\right|_{N^{\prime}} \in \varphi\left(r_{\left(N^{\prime}, x\right)}(\mathcal{E})\right)$.

\section{Results}

The main theorem of this paper is the following.

Theorem 1 If a correspondence $\varphi$ is consistent and unanimous, then $\varphi$ is a subcorrespondence of the Pareto correspondence.

The proof of Theorem 1 will be provided in Section 3. By this result, all the foregoing characterizations using consistency and Pareto efficiency simultaneously (such as Ergin (2000), and Ehlers and Klaus (2005)) can be strengthen by replacing Pareto efficiency with unanimity.

Given Theorem 1, in the sequel we are going for characterizations of the Pareto correspondence. Let us start with the following lemma.

Lemma 1 The Pareto correspondence is consistent and unanimous.

The proof of Lemma 1 is straightforward thus we omit it. Theorem 1 and Lemma 1 together yield the following characterization.

Theorem 2 The Pareto correspondence is the unique maximal correspondence that is consistent and unanimous.

Although Theorem 2 provides a characterization of the Pareto correspondence, this is not a full axiomatization of the correspondence. Here we will give a full axiomatization using "converse consistency." However, since the existing definition of converse consistency by Ergin (2000) does not work for this purpose, we have to modify the definition.

Ergin (2000) introduced the following definition of converse consistency: $\varphi$ is conversely consistent if for any problem $\mathcal{E}=(N, H, R)$ with $\# N \geq 2$, if for any $N^{\prime} \subset N$ with $\# N=2$, and any $x \in A(\mathcal{E}),\left.x\right|_{N^{\prime}} \in \varphi\left(r_{\left(N^{\prime}, x\right)}(\mathcal{E})\right)$, then $x \in \varphi(\mathcal{E})$. It is pointed out in Ergin's paper that the Pareto correspondence is not conversely consistent. 
For our purpose, we have to weaken Ergin's definition in the following way. Let $\mathcal{U}$ denote the correspondence defined as follows: For any $\mathcal{E}=(N, H, R)$,

$$
\mathcal{U}(\mathcal{E}):= \begin{cases}\mathcal{U}^{0}(\mathcal{E}) & \text { if } \mathcal{U}^{0}(\mathcal{E}) \neq \emptyset \\ A(\mathcal{E}) & \text { otherwise }\end{cases}
$$

$\varphi$ is $\mathcal{U}$-conversely consistent if for any problem $\mathcal{E}=(N, H, R)$ with $\# N \geq 2$, if for any $N^{\prime} \subset N$ with $\emptyset \neq N^{\prime} \neq N$, and any $x \in A(\mathcal{E}),\left.x\right|_{N^{\prime}} \in \varphi\left(r_{\left(N^{\prime}, x\right)}(\mathcal{E})\right)$ and $x \in \mathcal{U}(\mathcal{E})$, then $x \in \varphi(\mathcal{E})$.

In defining $\mathcal{U}$-converse consistency, we have made two modifications to Ergin's existing version: First, the present definition considers the reductions not only to pair subsets of the grand coalition but to all the nonempty proper subsets. Second, we condition that the allocation in consideration has to be a unanimous allocation as long as such an allocation is available. The first point is a natural weakening. The second point looks somewhat artificial. However, similar variations are used elsewhere such as Peleg and Tijs (1996), Peleg, Potters and Tijs (1996) and Takamiya (2001). ${ }^{1}$

Now we present a full axiomatization of the Pareto correspondence.

Theorem 3 A correspondence $\varphi$ is the Pareto correspondence if, and only if, $\varphi$ is consistent, unanimous and $\mathcal{U}$-conversely consistent.

The proof of this theorem is provided in Section 3. The tightness of this characterization is checked by the examples as follows.

Example 1 The correspondence $\mathcal{U}$ is unanimous and $\mathcal{U}$-conversely consistent, but not consistent.

In Example 1, it is easy to see that $\varphi$ violates consistency. Less obvious is to see that $\varphi$ is $\mathcal{U}$-conversely consistent. This follows from Lemma 4 which comes up shortly.

Example 2 Let $\varphi$ be such that for any $\mathcal{E}$,

$$
\varphi(\mathcal{E})=A(\mathcal{E})
$$

Then $\varphi$ is consistent and $\mathcal{U}$-conversely consistent, but not unanimous.

Example 3 Let $\succ$ be a linear ordering over $\mathcal{N}$. Let $\varphi$ be such that for any $\mathcal{E}=(N, H, R)$,

$$
\begin{array}{r}
i_{1}=\max \succ(N), \varphi\left(i_{1}\right)=\left\{\max R^{i_{1}}(H)\right\} ; \text { and } \\
\forall k=2, \ldots, \# N, i_{k}=\max \succ\left(N \backslash\left\{i_{1}, \ldots i_{k-1}\right\}\right), \\
\varphi\left(i_{k}\right)=\left\{\max R^{i_{k}}\left(H \backslash \varphi\left(\left\{i_{1}, \ldots, i_{k-1}\right\}\right)\right)\right\} .
\end{array}
$$

In words, $\varphi$ is the serial dictatorship with respect to the fixed order $\succ$. Then $\varphi$ is consistent and unanimous, but not $\mathcal{U}$-conversely consistent.

\footnotetext{
${ }^{1}$ One can think of two other versions of converse consistency: One is the version in which only the first modification is made to Ergin's definition. The other is the one in which only the second modification is made. We note that the Pareto correspondence does not satisfy either of these versions of converse consistency thus they do not work for our purpose.
} 


\section{Proofs of the main theorems}

This section provides the proofs of Theorems 1 and 3 . Although this paper studies the case of collective initial endowments only, in the following proofs, it will be helpful to consider the case of private initial endowments, too. Let a problem $\mathcal{E}=(N, H, R)$ be given. And let $\omega \in A(\mathcal{E})$. Then a house allocation problem with private initial endowments is $(\mathcal{E}, \omega)=(N, H, R, \omega){ }^{2}$

Let a problem $(\mathcal{E}, \omega)=(N, H, R, \omega)$ be given. Let $x, y \in A(\mathcal{E})$ and $S \subset N$. Then $x$ dominates $y$ via $S$ if the followings are satisfied:

$$
\begin{array}{r}
x(S)=\omega(S), \\
\forall i \in S, x(i) R^{i} y(i), \\
\exists j \in S: x(j) P^{j} y(j) .
\end{array}
$$

The core of $(\mathcal{E}, \omega)$ is the set of allocations that are not dominated by any allocations. It is well-known that the core is singleton for each preference profile by the result of Roth and Postlewaite (1977). ${ }^{3}$ Let us denote the single element of the core of $(\mathcal{E}, \omega)$ by $\mathcal{C}(\mathcal{E}, \omega)$.

There is a well-known algorithm called the "top trading cycle algorithm," which is attributed to David Gale (Shapley and Scarf, 1974), for computing the unique core allocation for each preference profile. We will utilize this algorithm in the proofs in the sequel. This algorithm works as follows:

(1) Let every agent $i$ point out the agent $j$ who has the object (as the initial endowment) that ranks top to the agent $i$. Let us denote this by " $i \rightarrow j$." Then there will be at least one cycle $i_{1} \rightarrow i_{2} \rightarrow \cdots \rightarrow i_{k} \rightarrow i_{k+1}$ with $i_{k+1}=i_{1}$. Let $T_{1}^{1}, T_{2}^{1}, \ldots, T_{M_{1}}^{1}$ denote the cycles formed at this stage. Let all agents in these cycles allocate the objects so that if $i \rightarrow j$, then $i$ receives the object $\omega(j)$.

(2) Let the agents in the $T_{1}^{1}, T_{2}^{1}, \ldots, T_{M_{1}}^{1}$ leave the scene taking away the objects allocated in the previous stage. And let the remaining agents do the same thing as (1) with the remaining objects to obtain the cycles $T_{1}^{2}, T_{2}^{2}, \ldots, T_{M_{2}}^{2}$.

(3) Repeat the process recursively until all the objects have been allocated. Then the set of cycles $\mathcal{T}:=\left\{T_{1}^{k}, T_{2}^{k}, \ldots, T_{M_{k}}^{k}\right\}_{k=1, \ldots, K}$ is obtained.

In the subsequent proofs, we will use the following two lemmas. We omit the proofs of these lemmas. The proof of Lemma 3 would be straightforward from the algorithm.

Lemma 2 For any $\mathcal{E}, \omega \in \mathcal{P}(\mathcal{E})$ if, and only if, $\omega=\mathcal{C}(\mathcal{E}, \omega)$.

Lemma 3 Let $(\mathcal{E}, \omega)=(N, H, R, \omega)$ be given. Let $\mathcal{T}$ be the top trading cycles corresponding to the core of $(\mathcal{E}, \omega)$. For any $T \in \mathcal{T}$, and any $i \in T, \mathcal{C}(\mathcal{E}, \omega)(i)=$ $\max R^{i}(\omega(T))$.

\footnotetext{
${ }^{2}$ We avoid the notation $((N, H, R), \omega)$, which is accurate but more cumbersome.

${ }^{3}$ Recall that we have assumed that a preference profile is a linear ordering. This result does not hold for the case in which weak preferences (ties) are permitted.
} 
Proof of Theorem 1 Let $\varphi$ be a correspondence. Suppose that $\varphi$ is consistent and unanimous, and that for some problem $\mathcal{E}=(N, H, R), \varphi(\mathcal{E}) \not \subset \mathcal{P}(\mathcal{E})$. Then there is some $x \in A(\mathcal{E})$ such that $x \in \varphi(\mathcal{E})$ and $x \notin \mathcal{P}(\mathcal{E})$. By Lemma $2, x \notin \mathcal{P}(\mathcal{E})$ implies

$$
x \neq \mathcal{C}(\mathcal{E}, x)
$$

Let us denote by $\mathcal{T}$ the top trading cycles associated with $\mathcal{C}(\mathcal{E}, x)$. Let us denote $y=\mathcal{C}(\mathcal{E}, x)$. Then there is some coalition $T \in \mathcal{T}$ such that

$$
\left.x\right|_{T} \neq\left. y\right|_{T}
$$

Since $T$ is a trading cycle arising from $x$,

$$
x(T)=y(T) .
$$

Thus $r_{(T, x)}(\mathcal{E})=r_{(T, y)}(\mathcal{E})$.

Since $y$ is the core allocation of $(\mathcal{E}, x)$, Lemma 3 implies $y(i)=\max R^{i}(x(T))$ for all $i \in T$. Then by the unanimity of $\varphi$,

$$
\left\{\left.y\right|_{T}\right\}=\varphi\left(r_{(T, x)}(\mathcal{E})\right) .
$$

On the other hand, since $\varphi$ is consistent, $\left.x\right|_{T} \in \varphi\left(r_{(T, x)}(\mathcal{E})\right)$. Therefore,

$$
\left.x\right|_{T}=\left.y\right|_{T} .
$$

However, this contradicts (1).

Next, we turn to the proof of Theorem 3. The proof is done by two steps. First, we will check that the Pareto correspondence is $\mathcal{U}$-conversely consistent. (We have already known that the correspondence satisfies the other two axioms.) Second, we will show the uniqueness: By Theorem 1, we have already known that if a correspondence $\varphi$ is consistent and unanimous, then $\varphi \subset \mathcal{P}$. We will show that $\varphi$ is $\mathcal{U}$-converse consistent and unanimous, then $\varphi \supset \mathcal{P}$. These entail $\varphi=\mathcal{P}$.

Lemma 4 Let a correspondence $\varphi$ be unanimous. Then if $\varphi$ is a supercorrespondence of the Pareto correspondence, then $\varphi$ is $\mathcal{U}$-conversely consistent.

Proof Suppose that $\varphi \supset \mathcal{P}$. Let $\mathcal{E}$ and $x \in A(\mathcal{E})$ be given. Suppose that $x \notin \varphi(\mathcal{E})$. Then we show either of the following is true:

$$
\exists N^{\prime} \subset N: N^{\prime} \neq\left. N \& x\right|_{N^{\prime}} \notin \varphi\left(r_{\left(N^{\prime}, x\right)}(\mathcal{E})\right),
$$

or

$$
x \notin \mathcal{U}(\mathcal{E}) .
$$

Denote $\mathcal{C}(\mathcal{E}, x)$ by $y$. Since $\varphi \supset \mathcal{P}$ by supposition, we have $x \notin \mathcal{P}(\mathcal{E})$. Then by Lemma $2, x \neq y$. Let $\mathcal{T}$ denote the top trading cycles associated with $y$. Since 
$x \neq y$, there is some $T \in \mathcal{T}$ such that $\left.x\right|_{T} \neq\left. y\right|_{T}$. And since $T$ is a trading cycle arising from $x, x(T)=y(T)$. Then Lemma 3 implies

$$
\left.x\right|_{T} \notin\left\{\left.y\right|_{T}\right\}=\mathcal{U}^{0}\left(r_{(T, x)}(\mathcal{E})\right) .
$$

Recall that $\varphi$ is unanimous. Then if $T \neq N$, then (4) implies (2). If $T=N$, (4) implies (3).

Lemma 5 Let a correspondence $\varphi$ be $\mathcal{U}$-conversely consistent and unanimous. Then $\varphi$ is a supercorrespondence of the Pareto correspondence.

Proof Let $\varphi$ be a correspondence. Suppose that $\varphi$ is $\mathcal{U}$-conversely consistent and unanimous, and that $\varphi \not \supset \mathcal{P}$. Then there is some $\mathcal{E}=(N, H, R)$ such that $x \in \mathcal{P}(\mathcal{E})$ and $x \notin \varphi(\mathcal{E})$. Then since $\varphi$ is $\mathcal{U}$-conversely consistent, $x \notin \varphi(\mathcal{E})$ implies either of the following is true:

$$
\exists N^{\prime} \subset N: N^{\prime} \neq\left. N \& x\right|_{N^{\prime}} \notin \varphi\left(r_{\left(N^{\prime}, x\right)}(\mathcal{E})\right),
$$

or

$$
x \notin \mathcal{U}(\mathcal{E}) .
$$

Suppose that $\mathcal{U}^{0}(\mathcal{E}) \neq \emptyset$. Then since $x \in \mathcal{P}(\mathcal{E}),\{x\}=\mathcal{U}^{0}(\mathcal{E})$. Thus the unanimity of $\varphi$ implies $\{x\}=\varphi(\mathcal{E})$. This is a contradiction. Therefore, we conclude that $\mathcal{U}^{0}(\mathcal{E})=\emptyset$. In this case, $\mathcal{U}(\mathcal{E})=A(\mathcal{E})$ by definition, which implies $x \in \mathcal{U}(\mathcal{E})$, the negation of (6). This implies (5) has to be satisfied.

Let $\mathcal{E}^{\prime}:=\left(N^{\prime}, H^{\prime}, R^{\prime}\right)$ denote the reduced problem $r_{\left(N^{\prime}, x\right)}(\mathcal{E})$ in $(5)$. Then since the above argument asserts (5) holds true, we have $\left.x\right|_{N^{\prime}} \notin \varphi\left(\mathcal{E}^{\prime}\right)$. Also by the fact that $\mathcal{P}$ is consistent (by Lemma 1 ), $\left.x\right|_{N^{\prime}} \in \mathcal{P}\left(\mathcal{E}^{\prime}\right)$. Thus we can apply the same argument as above to this case, too. Further, recursively applying this argument, we obtain some problem $\mathcal{E}^{\star}$ which consists of only one agent $i$, and satisfies $\left.x\right|_{\{i\}} \notin \varphi\left(\mathcal{E}^{\star}\right)$ and $\left.x\right|_{\{i\}} \in \mathcal{P}\left(\mathcal{E}^{\star}\right)$. However, the unanimity of $\varphi$ implies $\left.x\right|_{\{i\}} \in \varphi\left(\mathcal{E}^{\star}\right)$. This is a contradiction.

Proof of Theorem 3 By Lemmas 1 and 4, the Pareto correspondence is consistent, unanimous and $\mathcal{U}$-conversely consistent. Conversely, suppose a correspondence is consistent, unanimous and $\mathcal{U}$-conversely consistent. Then by Theorem 1 and Lemma 5, the correspondence has to coincide with the Pareto correspondence.

\section{References}

[1] Ehlers L and Klaus B (2005): Consistent house allocation. mimeo. Universite de Montreal. forthcoming in Econ Theory.

[2] Ergin Hİ (2000): Consistency in house allocation problems. J Math Econ 34: pp77-97.

[3] Peleg B, Potters JAM and Tijs SH (1996): Minimality of consistent solutions for strategic games, in particular for potential games. Econ Theory 7: pp81-93. 
[4] Peleg B and Tijs SH (1996): The consistency principle for games in strategic form. Int J Game Theory 25: pp13-34.

[5] Roth A and Postlewaite A (1977): Weak versus strong domination in a market with indivisible goods. J Math Econ 4: pp131-37.

[6] Shapley L and Scarf H (1974): On cores and indivisibility. J Math Econ 1: pp23-37.

[7] Takamiya K (2001): The consistency principle and an axiomatization of the $\alpha$-core. Int J Game Theory 30: pp195-207.

[8] Takamiya K (2006) Consistency and unanimity in the house allocation problems II: Private initial endowments. Mimeo. ISER, Osaka University.

[9] Thomson W (1996): Consistent allocation rules. University of Rochester Working Paper.

[10] Thomson W (1998): Consistency and its converse: An introduction. Rochester Center for Economic Research Working Paper, No. 448. 